\title{
EXTREME FIELD-OF-VIEW FOR HEAD-MOUNTED DISPLAYS
}

\author{
Ismo Rakkolainen ${ }^{*}$, Roope Raisamo ${ }^{*}$, Matthew Turk $^{+}$, Tobias Höllerer $^{+}$, Karri Palovuori $^{\S}$ \\ * University of Tampere, Faculty of Communication Sciences, 33014 Tampere, Finland \\ + University of California, Department of Computer Science, Santa Barbara, CA 93106, USA \\ $\S$ Tampere Univ. of Technology, Electronics and Communicat. Engineer., 33100 Tampere, Finland
}

\begin{abstract}
We present novel optics and head-mounted display (HMD) prototypes, which have the widest reported field-of-view (FOV), and which can cover the full human FOV or even beyond. They are based on lenses and screens which are curved around the eyes. While this is still work-in-progress, the HMD prototypes and user tests suggest a feasible approach to significantly expand the FOV of HMDs.
\end{abstract}

Index Terms - Head-mounted displays, field-of-view, virtual reality, $360^{\circ}$ video, 3D interaction, visualization

\section{INTRODUCTION}

Dioramas and stereoscopic image pairs have immersed the general public in imagery since the early 19 th century [28]. Numerous stereoscopic and 3D displays try to imitate reality [4].

Sutherland implemented the first head-mounted display (HMD) employing 3D graphics and head tracking [27]. HMDs are used to view synthetic computer graphics, virtual reality (VR) and cinematic, spherical $360^{\circ}$ (horizontal) $\times 180^{\circ}$ (vertical) video $\left(360^{\circ}\right.$ video). Such personal immersive displays have generated significant interest in the media and among the general public, and they have recently become feasible and affordable for consumer use. Smartphone-based VR viewers are increasingly used for VR.

A wide field-of-view (FOV) can improve the sense of immersion, presence and performance in some tasks in virtual environments. Currently popular HMDs have limited FOV (e.g., Oculus Rift $\sim 80^{\circ} \times 90^{\circ}$, HTC Vive $\sim 100^{\circ} \times 110^{\circ}$, Microsoft HoloLens $\sim 30^{\circ} \times 17^{\circ}$ ), even though there are some HMDs with wider FOV. The natural human FOV is still much wider $\left(\sim 210^{\circ} \times 135^{\circ}\right)$.

Usually both the lenses and the screens on an HMD are essentially flat. Wide-angle optics for flat displays presents a serious design challenge. Recent advances in display technology (e.g., flexible OLED) have reduced the problem of low resolution and they can bring also other new opportunities for HMDs.

Our contribution in this paper is to demonstrate improved super-wide FOV optical designs. We tested them with users, and also built functional proof-of-concept prototypes of super-wide FOV HMDs. One of them has the widest reported FOV.

As a base, we use the idea by Rakkolainen et al. [22], which employs curved screens and curved lenses around the eyes. It can use flexible OLED or other curved displays, and thin Fresnel lenses or holographic optical elements (HOE) as lenses. The prototypes presented in this paper show that the idea can be used for the peripheral areas of the human vision and for wide-FOV HMDs.

We first present issues of wide FOV and previous wideFOV HMDs, then present our HMD prototypes and user tests, discuss their limitations and potential improvements, and finally give some conclusions.

\section{WIDE FIELD-OF-VIEW}

The precision area of human vision (foveal vision) is just a few degrees wide, but as the gaze can move around, humans perceive as if it were larger. Most of the time humans direct gaze towards the front, not to the low-precision extreme sides (peripheral vision). Also, binocular vision, color vision, light sensitivity and the ability to perceive shape and motion vary across the visual field.

Wide-FOV displays convey peripheral information, improve orientation, situational awareness, object avoidance and performance in some tasks and are generally preferred by audiences. They also have an impact on perceptional issues, such as distance judgment, motion sickness, nausea and others [2], [16], [20], [13], [23], [24]. Even though super-wide FOV is generally conducive to simulator sickness, the FOV itself is not a problem, as the natural human FOV attests, but rather the insufficient implementation of current HMDs and conflicts of various sensory stimuli.

Interaction with VR and $360^{\circ}$ video content is limited and difficult [25], partly because the FOV is fairly limited. Full-surround display spanning the entire human FOV is an under-explored topic since there are currently few VR headsets that can support most of it. A recent study simulated HMD FOVs in user testing in the fully immersive, spherical Allosphere virtual environment. It shows that people complete tasks faster with full human FOV than with a limited FOV [24].

\section{PREVIOUS WIDE-FOV HMDS}

VR headset design consists of many trade-offs between optical and electronic elements and their impacts on HMD properties [9]. Many requirements and parameters for an HMD need to be balanced; e.g., wide FOV and high resolution are contradictory goals, as a wide FOV distributes the available pixels over a wider angle, reducing their spatial resolution. Other parameters are exit pupil size, latency, weight, price, etc.

Hundreds of HMDs (commercial and military products, academic prototypes and do-it-yourself devices by enthusiasts) have been published. Academic surveys show the multitude of the devices and various designs [7], [15]. Bungert [6] and Specout ${ }^{1}$ maintain lists of numerous past and present HMDs. HMDs are currently being developed by companies such as Microsoft, Facebook, Google, Sony, Samsung, Magic Leap, etc. Also multisensory HMDs have been proposed, e.g., mid-air tactile feedback [26], vibrotactile HMDs [18] and FeelReal VR Mask ${ }^{2}$.

LEEP optics [11] was the first feasible way to deliver wide FOV for HMDs. Since then several wide-FOV HMDs have been proposed, e.g., FakeSpace Wide $5^{3}$. Baek et al. [3] attached LCD displays to the peripheral areas of an HMD without any optics.

\footnotetext{
http://virtual-reality-headsets.specout.com/

2 http://feelreal.com/

${ }^{3}$ http://www.fakespacelabs.com/Wide5.html
} 
Oculus Inc. founder Palmer Luckey has built a $270^{\circ}$ FOV $\mathrm{HMD}^{4}$. Tiled displays merge numerous lenses and microdisplays seamlessly in order to increase FOV without reducing resolution [5], [8]. Other examples are $\mathrm{StarVR}^{5}$ and VRUnion Claire $12 \mathrm{M}^{6}$, which use flat Fresnel lenses for wide FOV.

Usually HMDs use flat screen and flat optics, but it is then difficult to obtain wide FOV. Some HMDs use curved mirrors or prisms (e.g., [14], [17]), but the image source is not curved. Roy$\mathrm{ole}^{7}$ uses flexible OLEDs on their HMDs. Wearality Inc. makes custom wide-FOV Fresnel lenses for smartphone VR viewers ${ }^{8}$. Rakkolainen et al. [22] has presented a super-wide optical design, which curves both lenses and screen seamlessly around the eyes (see Figure 1). Curved Fresnel lenses for HMDs are mentioned also in some patent applications, e.g., US2012/0120498, US2015/049390, WO2015/077718 and US2012/120498.

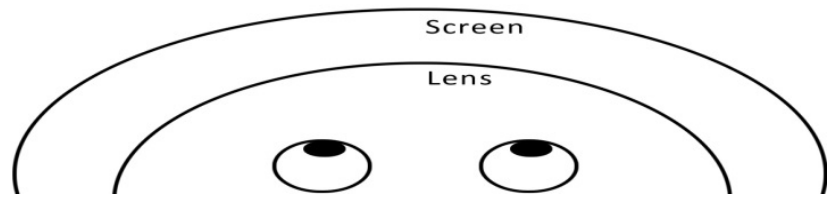

Figure 1. The basic idea of curved wide-FOV design [22]

Other approaches for wide-area vision are to compress a wide FOV image to fit it into the FOV of an HMD [1], [19] or to augment parts of it to the natural FOV [10], or to amplify the head movement [12].

\section{WORLD'S WIDEST FOV FOR HMD}

We explore further the feasibility of the curved Fresnel optics for HMDs [22]. A typical bulk Fresnel lens works as a flat magnifier for a flat surface. We however curved the lens around the eyes. The optimal curvature of the lens appears to be perpendicular towards the line of sight. As humans have two eyes, we made the Fresnel lenses to be fairly flat towards the front of the head, and more curved towards the sides. The side views do not need to be stereoscopic due to properties of the human vision.

For our super-wide-FOV prototypes, we used a stack of two bulk quality Fresnel lenses for each eye, which functioned promisingly. If the lenses of focal lengths $\mathrm{fl}$ and $\mathrm{f} 2$ are thin, the combined focal length $f$ of the stacked lenses is given by the basic lens equation:

$$
\frac{1}{\mathrm{f}}=\frac{1}{\mathrm{f} 1}+\frac{1}{\mathrm{f} 2}
$$

The lenses are made of flexible optical PVC plastic $(100 \times$ $89 \mathrm{~mm}, \mathrm{f}=120 \mathrm{~mm}$, groove pitch $0.3 \mathrm{~mm}$, thickness $0.4 \mathrm{~mm}$ ). The optical centers of the lenses were matched with forward direction of each eye. The eye distance from the Fresnel lens is about $25 \mathrm{~mm}$.

The VR mask has a nominal FOV of $232^{\circ} \times 130^{\circ}\left(266^{\circ}\right.$ diagonal), which exceeds the human FOV. The effective FOV is limited by the anatomy of human eye and head, as nose, cheeks, eyebrows, etc., block the most extreme peripheral views. Though the VR mask prototype is fairly compact, it is pragmatic to reduce the FOV, lenses and screens slightly for typical use.

The lens frame touches the skin (nose, eyebrow ridge and cheek). The weight of the lens construction is $40 \mathrm{~g}$. Figure 2 shows the super-wide-FOV curved Fresnel VR mask prototype without image planes.

\footnotetext{
${ }^{4} \mathrm{http}: /$ www.roadtovr.com/oculus-rift-creator-built-120-and-270-degree-fov-hmd-prototypes/

${ }^{5}$ http://www.starvr.com/

${ }^{6} \mathrm{http}: / /$ vrunion.com/

${ }^{7} \mathrm{http}: / / \mathrm{www} \cdot$ royole.com/

${ }^{8}$ http://www.wearality.com/wearalitysky/
}

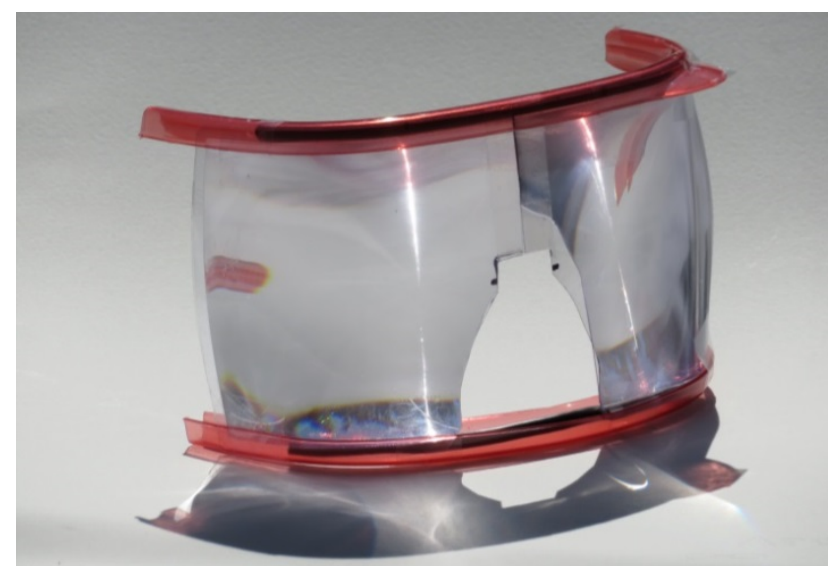

Figure 2. Super-wide-FOV curved Fresnel mask prototype

Fresnel lenses have a black-out effect that occurs if the viewing angle is too acute, but our prototype design avoids such extreme viewing angles. The far side areas of the screen have small optical distortions, but that is not an absolute impediment, as the human eye cannot see there easily or with high precision.

Flexible OLED displays, other curved displays, or projection with pico projectors would be good options for the curved image plane. Flexible OLED displays are not generally available at this point, and as our focus was on the feasibility of the optical idea, we simulated the curved image plane with images printed on curved paper or with three smartphones at $40^{\circ}$ angles (see Figure 5). They were in focus at about $45 \mathrm{~mm}$ away from the stacked lenses.

The image quality (resolution, colors, etc.) is well preserved in all directions, and the quality is promising, considering that this was a proof-of-concept prototype with little custom design and with low-cost bulk components. To illustrate the achieved image quality, we took photographs from the eye position through the lens towards various directions (see Figure 3).
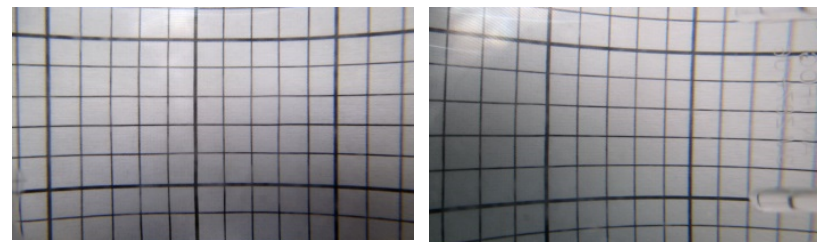

Figure 3. Pictures from the eye position towards front and $65^{\circ}$ to the side

The user cannot see precise images in the peripheral area, but this is more vision-limited than optics-limited. The screen resolution does not need to be high in the peripheral areas, which also eases requirements on peripheral rendering and optics. Even peripheral lights without any image can improve perception and reduce motion sickness [13], [29].

We tested the super-wide FOV with stereoscopic Cardboard VR apps on an LG G3 ${ }^{9}$ smartphone, which has a 5.5" screen with a resolution of $1440 \times 2560$ pixels $(515$ ppi). The smartphone screen and also the Fresnel lenses in the front are fairly flat. The stereoscopic images for each eye matched quite well and provided stereoscopy in the same way as with any VR viewer. The screen area at front near the nose appeared slightly out of focus for each eye, but the rest of it was in focus.

We also extended the side lenses (see Figure 4 left) and created an extreme $\mathrm{FOV}\left(318^{\circ} \times 130^{\circ} ; 343^{\circ}\right.$ diagonal $)$, which far exceeds the human FOV. It has the widest reported FOV, even though the excess beyond human FOV is not usable.

\footnotetext{
${ }^{9} \mathrm{http}: / /$ www.lg.com/us/mobile-phones/g3
} 

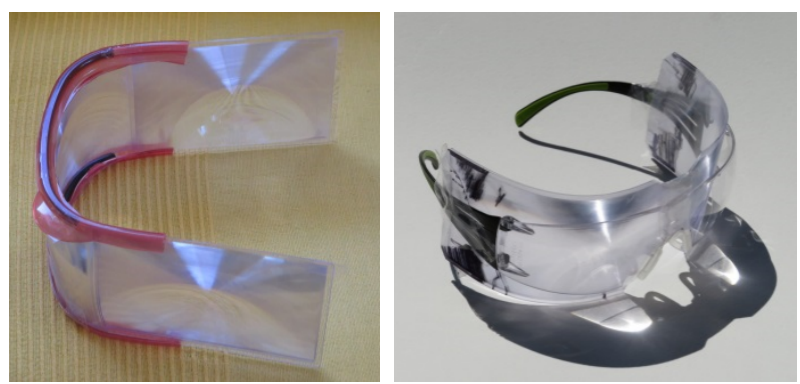

Figure 4. Left: World's widest FOV (343 diagonal). Right: Fresnel lenses curved around safety glasses (FOV: $293^{\circ}$ diagonal)

\section{USER EXPERIMENT OF OPTICS}

Rakkolainen et al. [22] made a legibility user test with their VR glasses. In order to compare our VR mask (Figure 2) and their glasses, we made similar VR glasses. We stacked two lenses $(114 \times 60 \mathrm{~mm})$ for each eye, and attached them to frameless safety glasses (see Figure 4 right). This FOV of $272^{\circ} \times 110^{\circ}$ exceeds the human horizontal FOV and is close to vertical FOV. We constructed varying optical prototypes, and the VR mask and the VR glasses were the most promising ones.

We conducted a user test with 10 participants (4 female, 6 male; $25-49$ years old; mean age: 37 ; normal vision). The image plane was a sheet of printed paper with random numbers at $8 \mathrm{pt}$ font size at $10^{\circ}$ intervals in two lines at the horizontal center. It was attached to a curved face-shield on a table and was well lit. With the optics in front of the eyes, we asked the participants to close the left eye and to position their head to the correct focus distance, with the right eye pointing directly to the center. They then read the numbers as far to the right as they could. Head rotation was not allowed, but eye rotation was allowed.

The participants could read the numbers up to about $35^{\circ}$. $60^{\circ}$ (mean $50^{\circ}$ ) to the side. There was some difference between the prototypes. The VR mask seemed to provide a slightly better legibility and clarity than the glasses. The participants could read the numbers with it in average $10^{\circ}$ wider to the side than with the glasses. All participants except two also had the opinion that the VR mask was either slightly or clearly better than the VR glasses in terms of optical clarity to all directions.

The specifications of the lenses on both the VR mask and the glasses are identical, apart from their size. One possible reason for the better perceived results of the VR mask may be due to some construction details such as the lens curvatures, which are slightly different between the VR mask and the glasses.

\section{A FUNCTIONING WIDE-FOV HMD}

We constructed a functioning HMD prototype. As lenses, we tested both the VR mask and the VR glasses, and approximated a curved screen by using three smartphones (LG G3), one in front and two on the sides at $40^{\circ}$ angles (see Figure 5). The flat image planes do not focus perfectly with the curved lenses, but they give an insight on the feasibility of the idea. The prototypes covered the full human horizontal visual field, but it has gaps of about $15^{\circ}$ between the front and peripheral smartphones due to the screen frames. Fresnel lenses are also prone to stray light, so an HMD frame should be covered. Internally, adjacent screens may cause some glare on the lenses if not properly designed.

The frontal image was fairly clear, but near the edges and the sides the screen was not always fully in focus, so the image was a little soft yet comprehensible on those areas. Due to the lens magnification only about half of the side screens were needed. For the side views of this prototype we used the half of stereoscopic rendering which was closer to the front display.

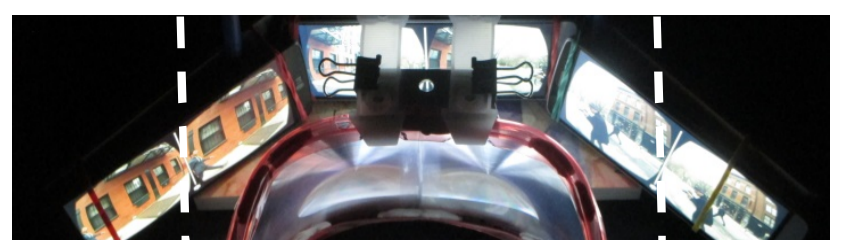

Figure 5. Curved wide-FOV optics with an approximation of a curved screen (using 3 smartphones with stereoscopic apps at $40^{\circ}$ angles). Only inner halves of the side screens were needed (visible).

We tested the prototype by viewing $360^{\circ}$ spherical videos. We employed a VR app Within (http://with.in/) set for Google Cardboard v2, and initially adjusted the images on the side smartphones by dragging them with finger so that all the three views matched together in static views. Even though the smartphones were not synchronized, the identical smartphones internally tracked their rotation movement in a coherent way for short periods of time and thus retained synchronized views.

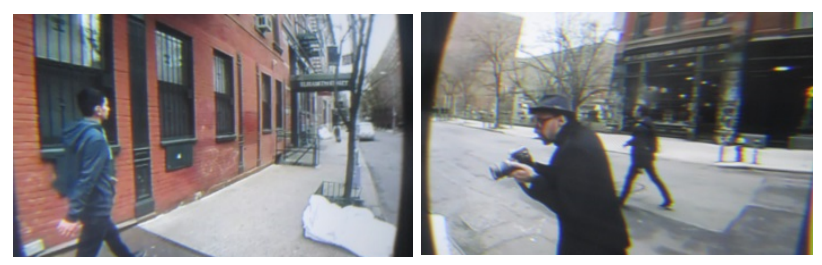

Figure 6. Pictures from the eye position towards front and $65^{\circ}$ to the side

The VR glasses provided visually better results than the VR mask, but this might be due to construction details and adjustments. Again, to illustrate the achieved image quality, we took photographs from the eye position through the Fresnel lens towards various directions (see Figure 6).

\section{DISCUSSION}

Several details can be improved in our proof-of-concept prototype. The construction is still fairly crude and could be more robust. Also image warping for the optics was not used. One of its limitations is that we approximated the curved image plane with printed paper (not a functional HMD) or with three smartphones (planar screens, heavy weight). Flexible OLED displays can be curved, have high resolution and contrast, low response time, are suitable for VR and entering the display market. The curved HMD concept may thus become relevant in the near future.

Thin and flexible Fresnel lenses and OLED screens may enable integrated device structures for casual VR viewing, along the lines of Rakkolainen et al. [21], C1-Glasses ${ }^{10}$ or Speck PocketVR ${ }^{11}$. When a Fresnel lens is directly touching the screen, it is fully transparent with no optical magnification and little optical artifacts. However, pop-up, pull-out, or foldable side displays and lenses, or possibly a dual-screen clamshell smartphone could enable compact, embedded and wide-FOV VR viewers.

Preferably, the Fresnel lens would be optically custom-designed, which could improve image quality significantly. Fresnel lenses in general are not as precise as traditional lenses, but they seem to be suitable at least for the peripheral human vision. Fresnel lenses are also employed in some HMDs, e.g., HTC Vive.

The perceptual issues need to be taken into account and more user testing is needed. The various psychophysical effects of super-wide FOV provide interesting research opportunities.

Curvature could be applied also vertically (to form a spherical, parabolic, etc. optical surface), but manufacturing such lenses (and matching spherical screens) would become more difficult, even though free-form optics can nowadays be printed.

\footnotetext{
${ }^{10} \mathrm{http}: / /$ goggletech.net/

$11 \mathrm{http}: / /$ www.speckproducts.com/pocket-vr.html
} 


\section{CONCLUSIONS}

We have further explored a super-wide FOV optical design for HMDs. Our proof-of-concept prototypes can cover the full human FOV with high resolution and have the world's widest reported FOV. They are also fairly light-weight and compact. They produced satisfactory results, considering the low cost (optics costs under USD 1 for each eye) and bulk quality of the Fresnel lenses, and properties of the human eye and its peripheral vision.

The prototypes suggest that wide-FOV HMDs may become feasible with curved optical design and emerging flexible display technologies. We will continue to develop the hardware and software to test these issues further with better prototypes and larger user populations for various tasks.

Super-wide FOV for HMDs can enhance the user experience by making it more immersive. VR, AR and $360^{\circ}$ videos also enable new kinds of spatial 3D user interfaces. We conclude that the optical design of a wide FOV is an intriguing and promising option for HMDs.

\section{ACKNOWLEDGMENTS}

We thank all the colleagues and reviewers who provided helpful comments on this paper. This work is partially funded by the Finnish Funding Agency for Innovation (Tekes, decision 1444/ 31/2016) and by Alfred Kordelin Foundation.

\section{REFERENCES}

[1] J. Ardouin, A. Lécuyer, M. Marchal, C. Riant, and E. Marchand, "FlyVIZ: a novel display device to provide humans with $360^{\circ}$ vision by coupling catadioptric camera with hmd", in Proc. ACM symp. on Virtual reality software and technology (VRST'2012), pp. 41-44.

[2] K. Arthur, Effects of Field of View on Performance with Head-Mounted Displays. PhD thesis. Univ. of North Carolina at Chapel Hill, 2000.

[3] J. Baek, J. Jung, and G. Kim, "Head mounted display with peripheral vision", in Proc. 2005 Int. Conf. on Augmented Tele-existence, 282.

[4] P. Benzie, J. Watson, J. Surman, I. Rakkolainen, K. Hopf, H. Urey, V. Sainov, and C. von Kopylow, "3DTV displays: techniques and technologies”, IEEE T. Circ. Syst. Vid., Vol. 17, 11, pp. 1647-1658, Nov. 2007.

[5] L. Brown and Y. Boger, "Applications of the Sensics Panoramic HMD”, in Proc. of the SID Symp. Digest of Technical Papers vol. 39, 1, pp. 77-80.

[6] C. Bungert, "HMD/headset/VR-helmet comparison chart", http://www.stereo3d.com/hmd.htm. Accessed May 3, 2017.

[7] O. Cakmakci and J. Rolland, "Head-worn displays: a review”. J. of Display Tech., vol. 2, 3, pp. 199-216, 2006.

[8] D. Cheng, Y. Wang, H. Hua, J. Sasian, "Design of a wideangle, lightweight head-mounted display using free-form optics tiling", Opt. Lett. vol. 36, 11, pp. 2098-100, 2011.

[9] C. Chinnok, "Understanding Trade-offs in Microdisplay and Direct-View VR Headset Designs", Insightmedia report, February 2017, www.insightmedia.info.

[10] K. Fan, J. Huber, S. Nanayakkara, and M. Inami, "SpiderVision: extending the human field of view for augmented awareness", in Proc. 5th Augmented Human Int. Conf. (AH '14), 2014, Article 49.

[11] E. Howlett, "Wide angle orthostereo", in Proc. 1990 SPIE Conf. on Stereoscopic Displays and Applicat., pp. 210-223.
[12] C. Jay and R. Hubbold, "Amplifying Head Movements with Head-Mounted Displays", Presence, Vol. 12, 3, pp. 268276, June 2003.

[13] J. Jones, J. Swan, and M. Bolas, "Peripheral Stimulation and its Effect on Perceived Spatial Scale in Virtual Environments". IEEE Trans. on Visualization \& Comp. Graphics, 19, 4, pp. 701-710, March 2013.

[14] K. Kiyokawa, "A wide field-of-view head mounted projective display using hyperbolic half-silvered mirrors", in Proc. ISMAR'2007 Conf., pp. 1-4.

[15] B. Kress and T. Starner, "A review of head-mounted displays (HMD) technologies and applications for consumer electronics", in Proc. SPIE 8720, Photonic Applications for Aerospace, Commercial, and Harsh Environments IV, 87200A, May 2013.

[16] J. Lin, H. Duh, D. Parker, H. Abi-Rached, and T. Furness, "Effects of Field of View on Presence, Enjoyment, Memory and Simulator Sickness in a Virtual Environment", in Proc. IEEE Virtual Reality 2002, pp. 164-171.

[17] H. Nagahara, Y. Yagi, and M. Yachida, "Super wide viewer using catadioptrical optics", in Proc. ACM symp. on Virtual reality software and technology (VRST'2003), pp. 169-175.

[18] V. Oliveira, L. Nedel, A. Maciel, and L. Brayda, "Localized Magnification in Vibrotactile HMDs for Accurate Spatial Awareness", in Proc. Eurohaptics 2016 Symp., pp. 55-64.

[19] J. Orlosky, Q. Wu, K. Kiyokawa, H. Takemura, and C. Nitschke, "Fisheye vision: peripheral spatial compression for improved field of view in head mounted displays", in Proc. 2nd ACM symp. on Spatial user interaction (SUI'2014), pp. 54-61.

[20] R. Patterson, M. Winterbottom, and B. Pierce, "Perceptual Issues in the Use of Head-Mounted Visual Displays". $\mathrm{Hu}$ man Factors 48, 3, pp. 555-573, 2006.

[21] I. Rakkolainen, R. Raisamo, M. Turk, T. Höllerer, and K. Palovuori, "Casual Immersive Viewing with Smartphones", in Proc. 20th Int. Acad. Mindtrek Conf. 2016, pp. 449-452.

[22] I. Rakkolainen, M. Turk, and T. Höllerer, "A Super-wideFOV Optical Design for Head-Mounted Displays", in Proc. Joint 26th Int. Conf. on Artificial Reality and Telexistence \& the 21th Eurographics Symp. on Virtual Environments (ICAT-EGVE 2016), pp. 45-48.

[23] C. Rash, M. Russo, T. Letowski, and E. Schmeisser, Helmet-Mounted Displays: Sensation, Perception and Cognition Issues. U.S. Army Aeromedical Research Lab, 2009.

[24] D. Ren, T. Goldschwendt, Y. Chang and T. Höllerer, "Evaluating wide-field-of-view Augmented Reality with Mixed Reality Simulation", in Proc. IEEE VR'2016, pp. 93-102.

[25] G. Rovelo, D. Vanacken, K. Luyten, F. Abad, and E. Camahort, "Multi-viewer gesture-based interaction for omnidirectional video", in Proc. ACM CHI'14, pp. 4077-4086.

[26] A. Sand, I. Rakkolainen, K. Palovuori, P. Isokoski, J. Kangas, and R. Raisamo, "Head-Mounted Display with Mid-Air Tactile Feedback", in Proc. ACM Symp. on Virtual Reality Software and Technology (VRST 2015), pp. 51-58.

[27] I. Sutherland, "A Head-mounted Three-dimensional Display", in Proc. of the AFIPS Fall Joint Computer Conf. 1968, Thompson Books, Vol. 3, pp. 757-764.

[28] C. Wheatstone, "On Some Remarkable, and Hitherto Unobserved, Phenomena of Binocular Vision", Philosoph. Trans. of the Royal Soc. of London, vol. 11, pp. 371-394, 1838.

[29] R. Xiao and H. Benko, "Augmenting the Field-of-View of Head-Mounted Displays with Sparse Peripheral Displays", in Proc. ACM CHI 2016 Conf., pp. 1221-1232. 